
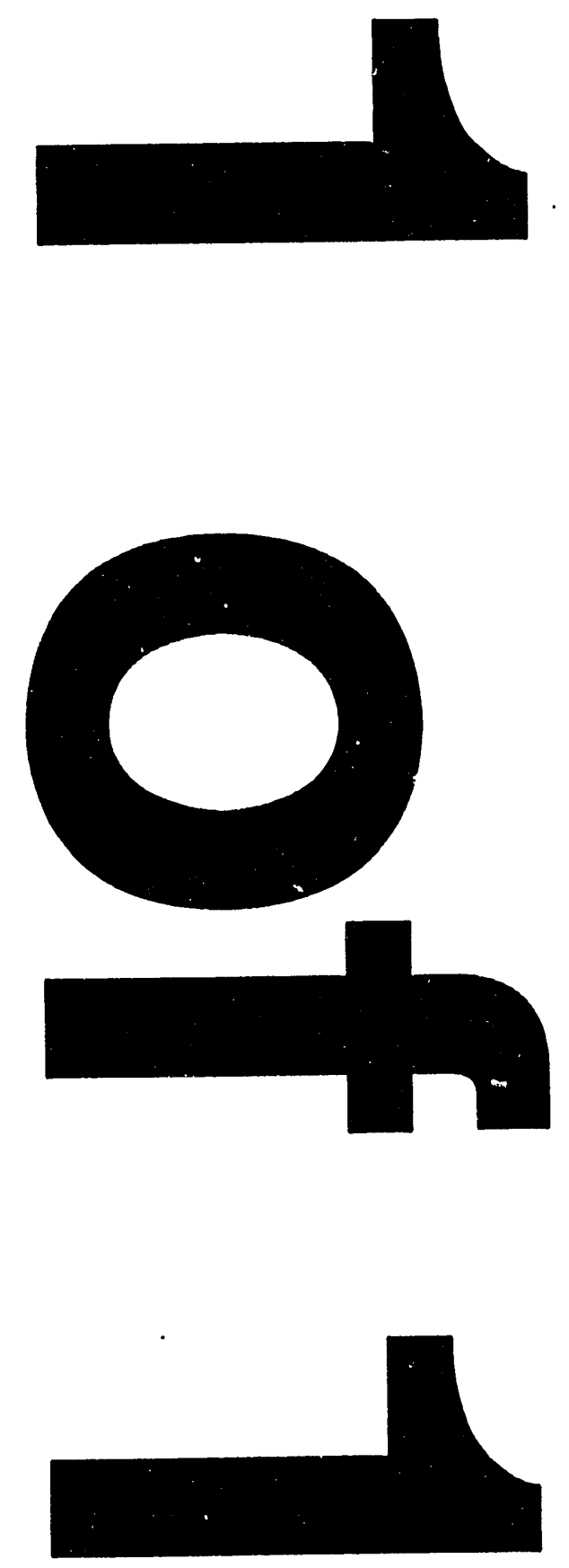


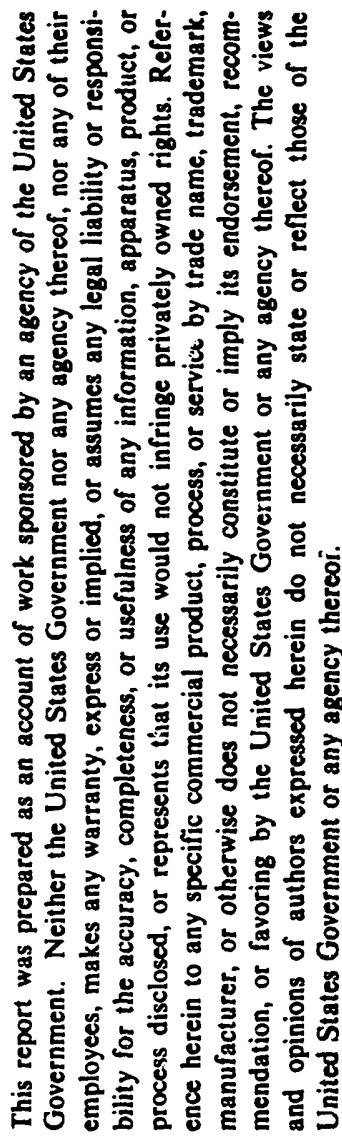

Title:

A SECOND REPORT ON THE EXPERIMENTAL PROGRESS OF THE ONE MICROSECOND, ONE RILOJOULE PER PULSE L-BAND

Submitted to:

Author(s): M. V. Fazio, T. J. Kwan, and R. M. Stringfield
OE, LASE 194, LOS Angeles, CA

January 22-28, 1994

\section{Los Alamos}

NATIONAL LABORATORY

\section{MASTER}

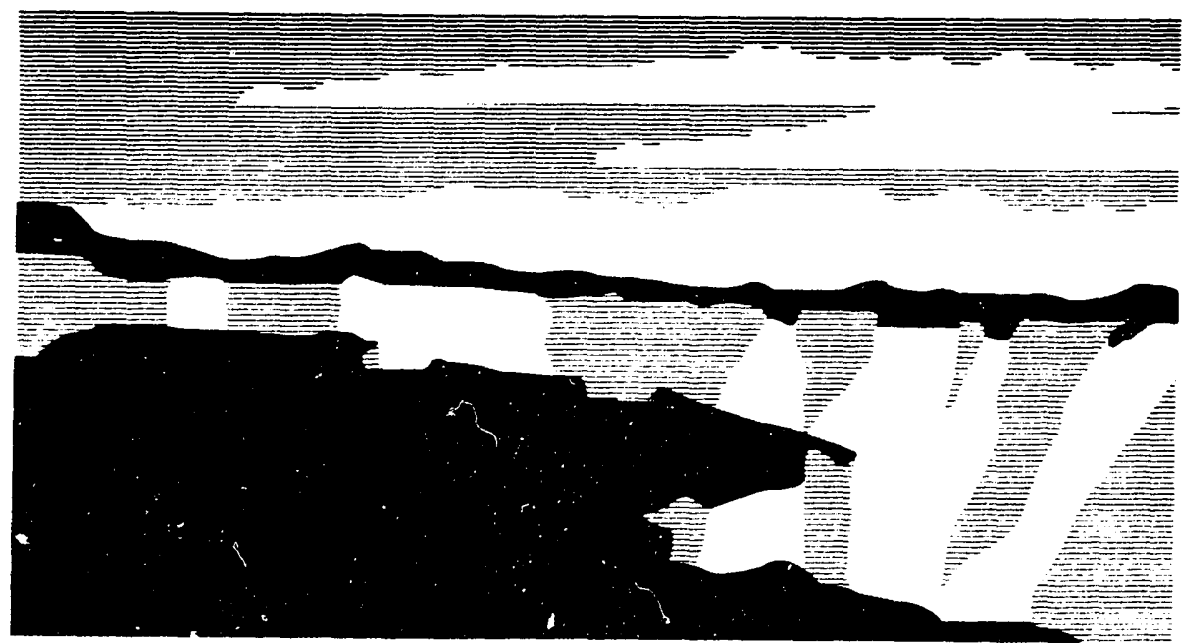

Los Alamos National Laboratory, an affirmative action/equal opportunity empłdyer, is operated by the University of California for the U.S. Department of Energy under contract W-7405-ENG-36. By acceptance of this article, the publisher recognizes that the U.S. Government retains a nonexclusive, royalty-free license to publish or reproduce the published form of this contribution, or to allow others to do so, for U.S. Government purposes. The Los Alamos National Laboratory requests that the publisher identify this article as work peiformed under the auspices of the U.S. Department of Energy. 


\title{
A Second Report on the Experimental Progress of the One Microsecond, One Kilojoule per Pulse L-Band Relativistic Klystron at Los Alamos
}

\author{
W.B. Haynes, B.E. Carlsten, R.J. Faehl, M.V. Fazio, T.J.T. Kwan, R. M. Stringfield \\ Los Alamos National Laboratory, MS H851, Los Alamos, NM 87545
}

\begin{abstract}
Work is continuing on a high-current relativistic kiystron amplifier (RKA) with the goal of producing $1 \mathrm{~kJ}$ per pulse with a $1 \mu$ s pulsewidth and a peak power of $1 \mathrm{GW}$. The three cavity tube has already produced pulses with more than $150 \mathrm{~J}$ and over 450 MW peak power. The original output cavity was thought to be limiting the performance, and a new cavity has been designed, built, and is now on-line and being conditioned up towards high power. Current experimental results are presented.
\end{abstract}

\section{INTRODUCTION}

Los Alamos is developing an L-band high current relativistic klystron. Although the present experiments are single pulse, the long term goal is to achieve $1 \mathrm{~kJ} /$ pulse with repetitive pulse capability at a PRF of $5 \mathrm{~Hz}$ with a longer term goal of $100 \mathrm{~Hz}$. The RKA has an input cavity, a single idler cavity, and an output cavity. The buncher section, which consists of the input and idler cavities, has been experimentally tested and appears to be performing as designed. The design was done with the help of particle-in-cell (PIC) code calculations using the Los Alamos code ISIS. PIC code modeling has proven to be very important for successful design because of the highly nonlinear nature of the RKA caused by the intense space charge effects ${ }^{1}$. The most recent efforts involve redesigning the output cavity of the tube and experimentally optimizing the output power and pulse length to reach the design goal of $1 \mathrm{~kJ} /$ pulse. A number of expected problems have been encountered with the output cavity that are being systematically addressed. These problems include if breakdown in the cavity and matching the beam impedance to the cavity gap shunt impedance for the most efficient coupling of modulated beam to microwaves. This paper summarizes the performance of the idler cavities and the modified output cavity and presents the data that has been taken with the latest output cavity.

\section{EXPERIMENTAL WORK}

The RKA in its current configuration is shown schematically in Fig. 1. 


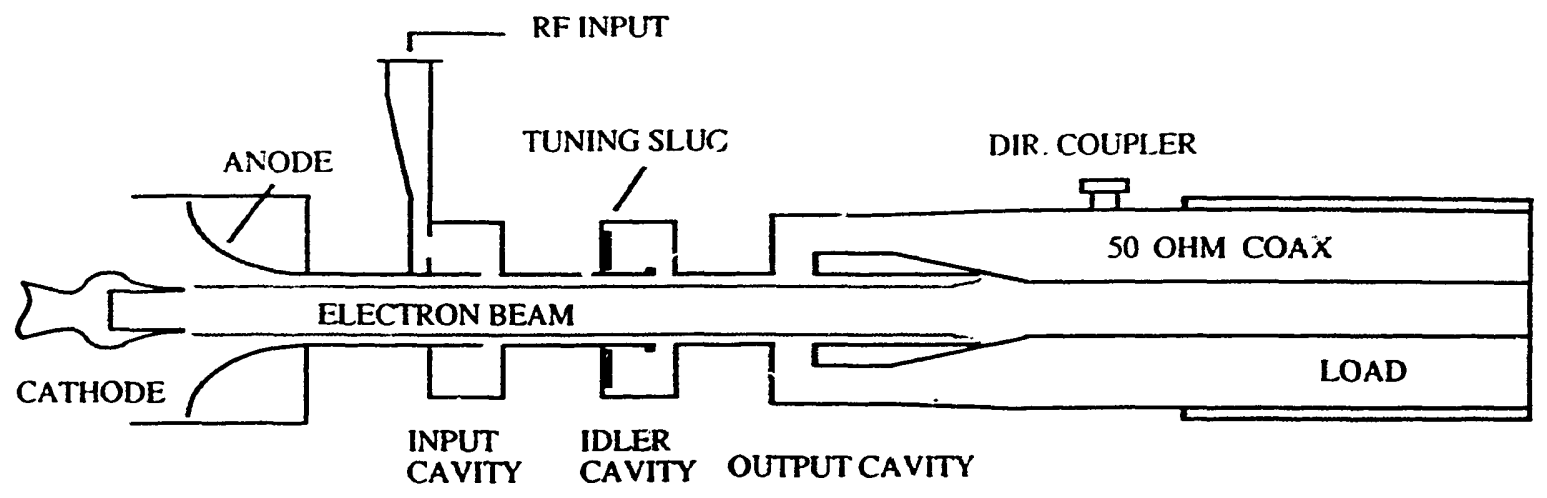

Fig. 1: Schematic diagram of the RKA

The electron beam is formed from a $6.2 \mathrm{~cm}$-diameter annular field-emission cathode and is slightly compressed by a converging $0.5 \mathrm{~T}$ axial magnetic field to a nominal $5.9 \mathrm{~cm}$ diameter beam with a $5 \mathrm{~mm}$ thickness. The typical beam voltage is $620 \mathrm{kV}$ with an increasing current of 3-6 kA caused by the drop in gun impedance during the pulse due to plasma closure of the anode-cathode gap. The input and idler cavities are of the quarterwave coaxial geometry type. The input cavity is coupled through an iris into reducedheight WR-650 waveguide that tapers to full height and connects to a $300 \mathrm{~kW}$ L-band magnetron. The idler cavity has an annular tuning ring which gives flexibility in inductively tuning the cavity. The output cavity is a noseless pillbox design with an annular coupling slot near the outer diameter which couples power into a low impedance coaxial transmission line. The coax tapers out into standard 6-inch-diameter $50 \Omega$ line where power is measured by a directional coupler with $15 \mathrm{~dB}$ directivity and then dissipated in a dummy load. The performance of the input cavity was detailed at last year's conference and will not be repeated here?

\subsection{Idler cavity}

The role of the idler cavity is to substantially increase the beam modulation that is initiated by the input cavity. The idler cavity is placed at the position downstream of the input cavity where the beam modulation reaches its highest valuc, which is where $I_{1} / I_{0}$ is about $10 \%$. The idler cavity can be inductively tuned over about $100 \mathrm{MHz}$, from 1300 to $1400 \mathrm{MHz}$. Conventional klystrons use several idler cavities and can afford to sacrifice individual cavity gain and yet still achieve a high beam modulation by tuning the cavities far off resonance. Since the RKA had only one idler cavity for these experiments, we require high gain and tune the idler close to the drive frequency. Measurements were made of beam harmonic current modulation $\left(\mathrm{I}_{1}\right)$ as the if input power was varied and as the idler cavity tuning was changed. The harmonic current clearly increases, as predicted, as the idler cavity tuning moves toward the $1.30 \mathrm{GHz}$ if drive frequency for a given amount of iinput drive. We could not adjust the original idler cavity frequency any closer than $1344 \mathrm{MHz}$ because the peak surface field at the idler cavity gap exceeded the level for $\mathrm{rf}$ breakdown. Rf breakdown across the idler cavity gap was encountered when the input drive power was increased in order to increase the beam modulation and when 
tuning the idler cavity near the magnetron drive frequency. The of breakdown caused a major disruption of the beam modulation.

We disassembled the idler cavity for visual inspection. The reentrant nose and the cpposite side of the gap showed strong evidence of arcing. To solve this problem the reentrant nose of the idler cavity was replaced by one with a much larger radius of curvature in order to reduce the peak surface field. The original reentrant nose was simply the rounded edge of the 0.065 -in-thick beam drift pipe. The modified nose had a radius of 0.1 in. The gap of the idler cavity was increased to oftset the increase in capacitance due to the larger radius nose, and bring the resonant frequency of the cavity back to near the original design.

Beam modulation data were taken for different idler tuning frequencies and input cavity drive powers to find the best combination for maximum beam modulation for the original idler cavity. The data showing beam modulation $\left(I_{1} / I_{0}\right)$, at the location of the output cavity gap, as a function of input drive power is plotted in Fig. 2

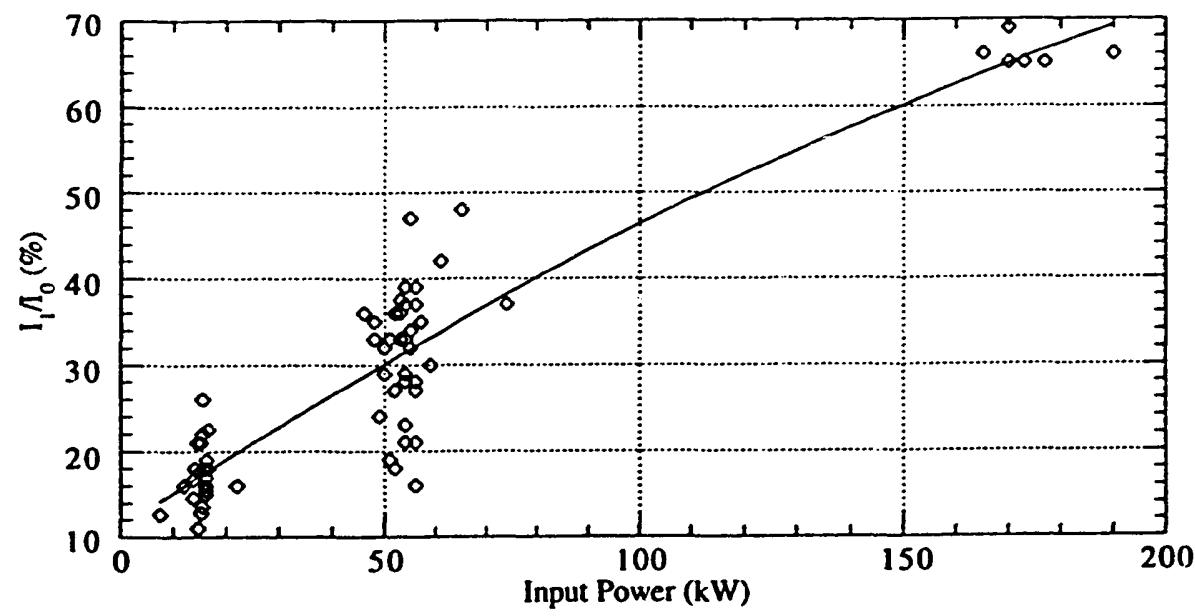

Fig. 2: Beam nodulation versus if input drive power

The data showing the beam modulation as a function of idler cavity tuning is somewhat incomplete because we installed the output cavity as soon as it was available.

From PIC code simulations, the amount of modulated current out of the idler cavity for maximum output was determined to be $65-75 \%^{3}$. In Fig. 3 , the beam current $\left(\mathrm{I}_{0}\right)$ and the detected signal $\left(I_{1}\right)$ from a B-dot probe located $22 \mathrm{~cm}$ downstream of the modified idler cavity prior to the installation of the output cavity are shown. 


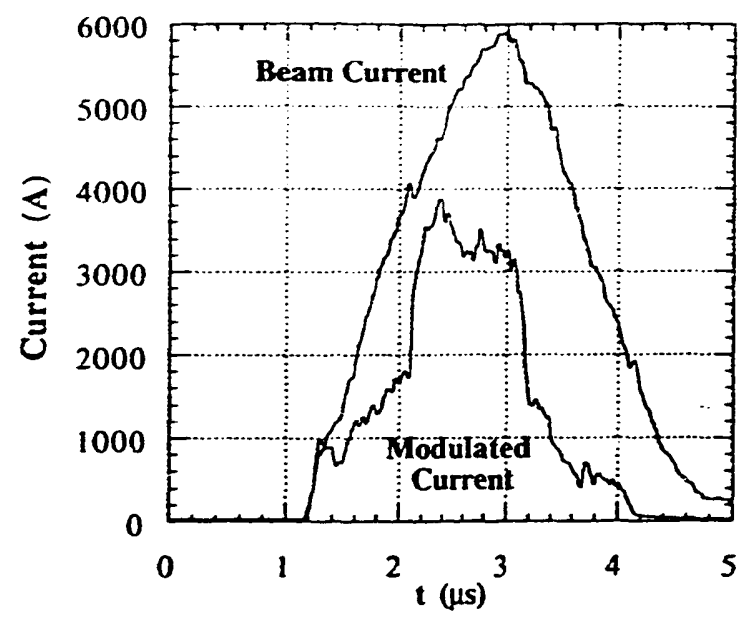

Fig. 3a: Beam Current $\left(\mathrm{I}_{0}\right)$ and Modulated Beam Current $\left(\mathrm{I}_{1}\right)$ for Shot 1516.

The modulation of the beam is over $60 \%$ for most of the pulse length. The amount of extractable beam power is usually given as $P=\left(V^{2} I_{1}\right) / 2$. For this case with an average voltage of $650 \mathrm{kV}$ and an average harmonic current of $3.2 \mathrm{kA}$, about $1 \mathrm{GW}$ of extractable power is in the fundamental harmonic. All of the kinetic energy associated with the modulated beam cannot be extracted because of the effect of the space charge potential depression ${ }^{3}$. Therefore, the output cavity can only extract about $70 \%$ of this power, and should produce $700 \mathrm{MW}$ of if output power or $700 \mathrm{~J}$ per pulse.

\subsection{Output Cavity Development}

\subsubsection{The Modified Output Cavity}

So far, the highest energy obtained with the modified output cavity, in single pulses, has been $160 \mathrm{~J}$. Data are shown in Fig. 4 where if output power, beam voltage, and beam current are all overlaid on the same time scale.

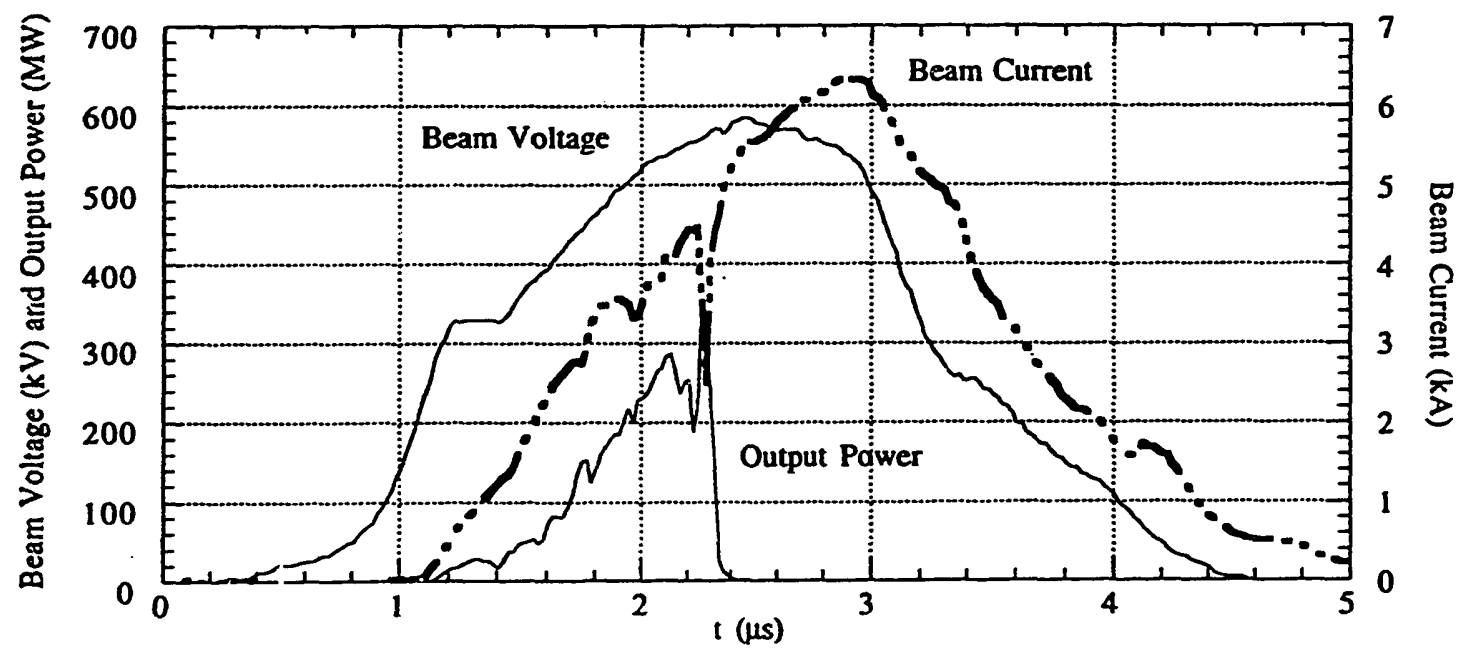

Fig. 4: Beam Voltage, Current, and Output Power for Shot 1308 
The peak power is $375 \mathrm{MW}$. Note that the if power goes away before the beam power reaches its maximum value. This will be discussed later. The frequency down-converted mixer IF signal from this shot, shown in Fig. 5, is relatively clean. The FFT of the mixer signal is shown on the right.
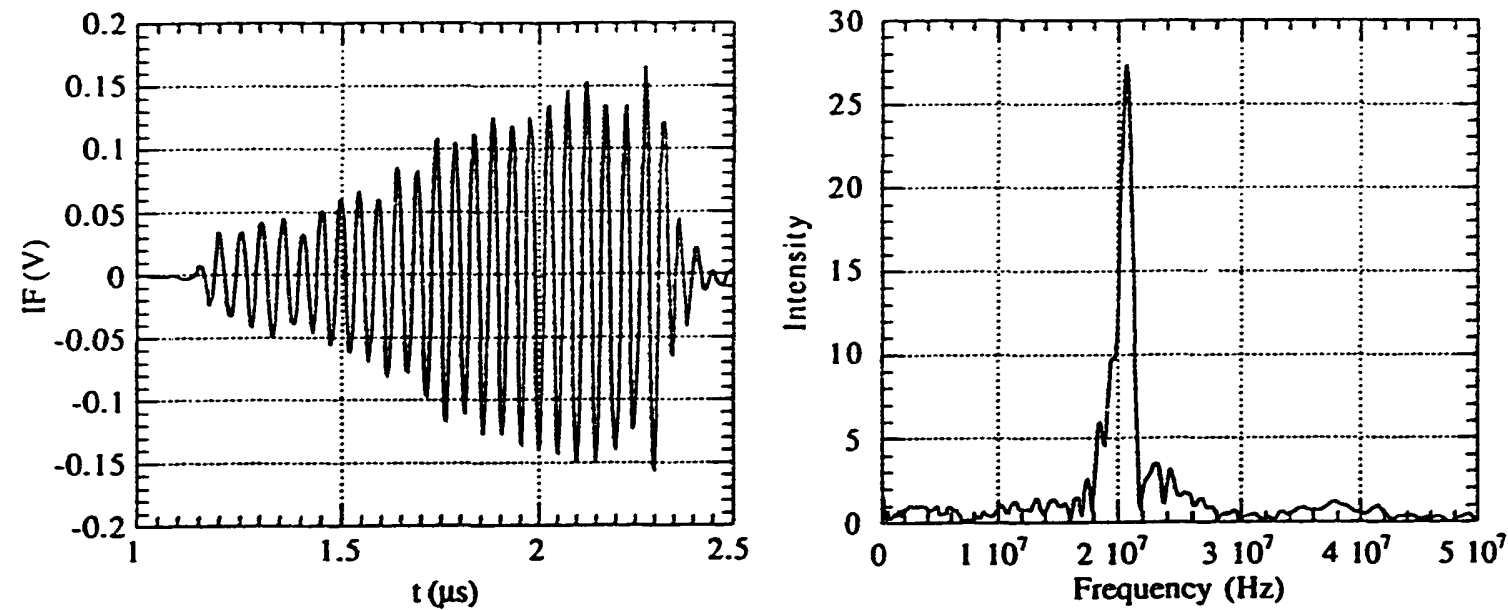

Fig. 5: Downconverted mixer signal from the output of Shot 1308, L.O. $=1280 \mathrm{MHz}$

The $3 \mathrm{~dB}$ bandwidth is $0.9 \mathrm{MHz}$. Other shots have recorded higher peak powers, although at a slightly lower energy per pulse. The signals from the two B-dot loops located 90 degrees apart on the upstream wall of the output cavity are shown in Fig. 6.

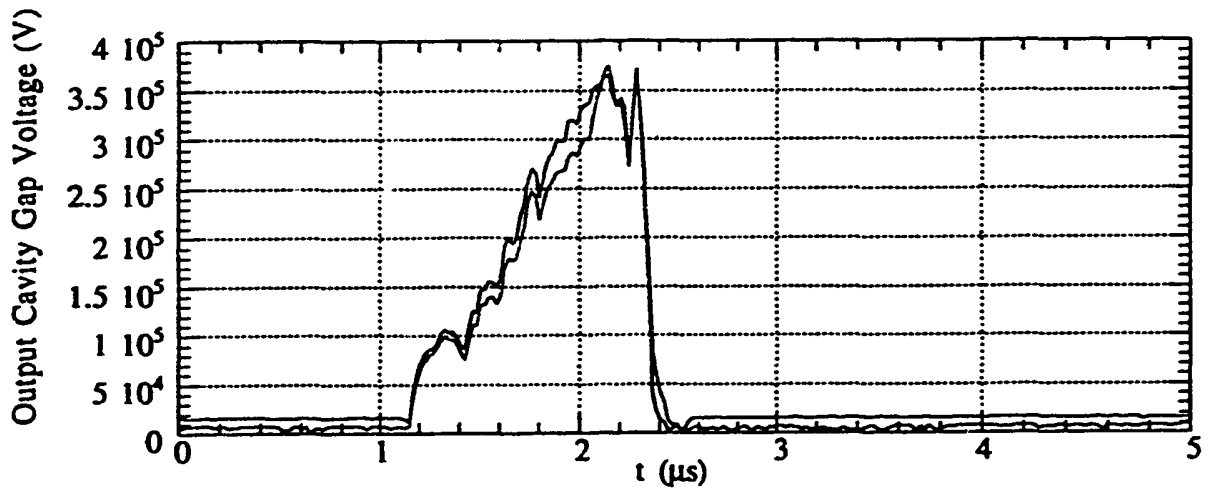

Fig. 6: Output Cavity gap voltage determined from two cavity B-dots

They track together indicating that the output cavity is operating in the proper mode. The B-dot signals are plotted in Fig. 6 in terms of the output cavity gap voltage they represent. The actual voltage across the $2.76 \mathrm{~cm}$ output gap at $375 \mathrm{MW}$ reaches $370 \mathrm{kV}$ producing an average electric field of about $134 \mathrm{kV} / \mathrm{cm}$. The output cavity has been modeled with a three-dimensional rf structures simulator program, known as the High Frequency Structure Simulator (HFSS) ${ }^{4}$. From the HFSS simulation, the magnitude of the peak gap voltage, if $500 \mathrm{MW}$ is being coupled into the output coax, was determined to be as high as $538 \mathrm{kV}$ across the geometrical cavity gap with a peak surface electric field of 225 $\mathrm{kV} / \mathrm{cm}$. This gap voltage was high enough to turn electrons around in the beam. 
Therefore, it was clear that the modified output cavity would never be able to reach the desired output power level of $1 \mathrm{GW}$.

At this point it is not yet certain why the rf pulse is terminating prematurely. The most reasonable explanation at this time is that the output cavity is producing fields high enough to reflect electrons back up the beam-line and/or start some oscillation phenomenon such as a virtual cathode. Fig. 7 shows traces representing magnetron forward and reflected power, the B-dot loop signal in the idler cavity, and the B-dot signal in the input cavity.

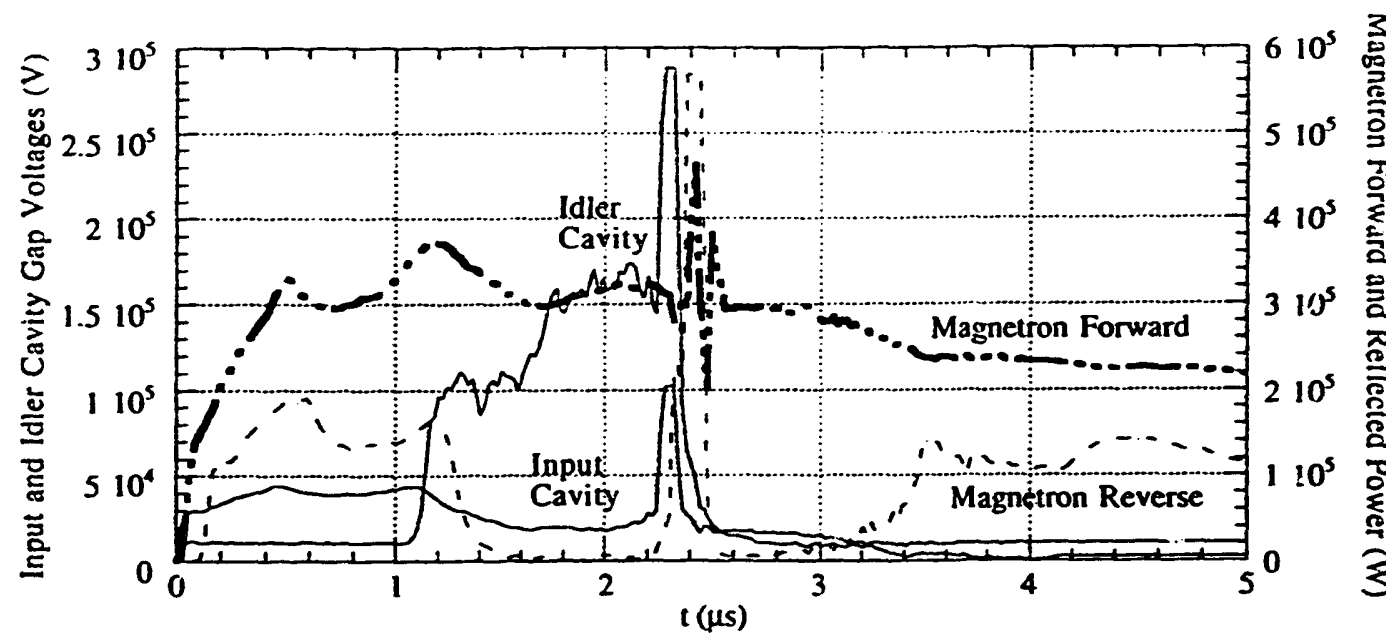

Fig. 7: Input and Idler Cavity Gap Voltages with Magnetron Power for Shot 1308

All of these signals have a spike at the time the if output power goes away. These signals are bandpass filtered with a bandwidth of several hundred $\mathrm{MHz}$ around $1.3 \mathrm{GHz}$, so the spike appears to be the result of an if modulation on the beam (space charge wave) traveling back up the beam toward the cathode. The interpretation of the observation is complicated by the fact that the output cavity gap appears to be breaking down at this time as well.

\subsubsection{New Output Cavity Design}

We realized, with the help of the ISIS simulations, that bunching the beam too strongly $\left(\mathrm{I}_{1} / \mathrm{I}_{0}>1\right)$ would result in an unacceptable loss of beam kinetic energy due to the increased potential depression associated with the much higher space charge of the fundamental harmonic current. Instead, the ideal beam modulation appeared to be about $65 \%$. To extract this energy efficiently requires an output cavity with a loaded $Q$ of about 4 , a factor of two higher than originally predicted. This is primarily because the harmonic beam impedance is lower at $65 \%$ modulation necessitating a lower output cavity shunt impedance for good conversion efficiency. A completely new output cavity with a lower loaded $Q$ has been designed using HFSS. The ability to do realistic 3-D electromagnetic modeling is proving to be critical for developing new, realistic output cavity designs. An output cavity is a true three dimensional problem with traveling wave boundary conditions. Other than HFSS, to our knowledge there is no other computational code available that can solve 3-D problems with traveling wave boundary conditions in the frequency domain, although MAFIA can address similar problems in the time 
domain. The new output cavity will have a lower output gap voltage, thereby reducing the probability for electron reflection and if breakdown across the gap. For an output power of $500 \mathrm{MW}$, the geometrical gap voltage is calculated to be $365 \mathrm{kV}$ and the peak surface field is $246 \mathrm{kV} / \mathrm{cm}$. The peak surface field has not increased substantially over the value in the modified original cavity. but the gap voltage has been drastically decreased. The peak surface field is what causes the of breakdown, while electron reflection is determined by the line integral of the electric field along the electron trajectory across the geometrical output gap and the fringing fields just inside the drift pipe flanking the gap. The lower gap voltage should allow the new cavity to consistently extract $0.5 \mathrm{GW}$ or more for the microsecond-long beam pulse.

The adjustable tuning, output loading, and gap length features in the original output cavity were not useful because the output loading could not be made strong enough, even by machining most of the vanes away to open the irises almost completely. The new design has dropped most of the adjustable features of the original design in favor of creating a cavity specialized for extracting the high modulated power present on the very low impedance beam. A very low cavity shunt impedance is necessary to efficiently couple to the low impedance beam.

The new pillbox-shaped cavity has a considerably smaller outside diameter which will make the cavity less likely to induce virtual cathode formation caused by the large space-charge bunches passing through the gap. The decreased $Q$ will help prevent the cavity fields from reaching the point where electrons are reflected. The coupling slots have been increased in both height and angular width. There is no variable coupling adjustment, and the tuning slug has also been eliminated. A separate tuning control is usually desirable, but the only available location would put the tuning slug in the position where the magnetic field is strongest (near the cavity's outer wall) and this is the location desired for the output coupling irises. The one adjustment that has been retained is the gap length, which can tune the frequency to some extent. Views of the new design are shown in Fig. 8.
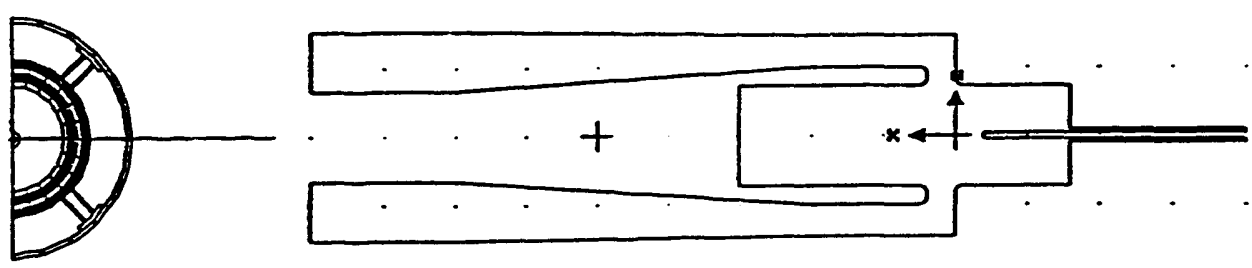

Fig. 8: Cross-sectional and end views of the new output cavity

The end view only shows one half of the structure because, due to symmetry, it was not necessary to model the complete structure. An electric-field probe has been added on the right side for modeling purposes to supply an input signal. The beam passes from right to left. The output cavity section is coupled to the low impedance coaxial line though 4 irises defined by 4 posts. The output coax then tapers to the standard 6-in-diam., $50 \Omega$ line dimensions. This cavity has a resonant frequency of $1300 \mathrm{MHz}$ and a loaded $\mathrm{Q}$ of about 4.1 according to HFSS. 
Cold test results from measuring the new cavity are shown in Figure 9. After smoothing the ripple from the B-Dot response, it is clear that th: cavity is oscillating essentially as designed.

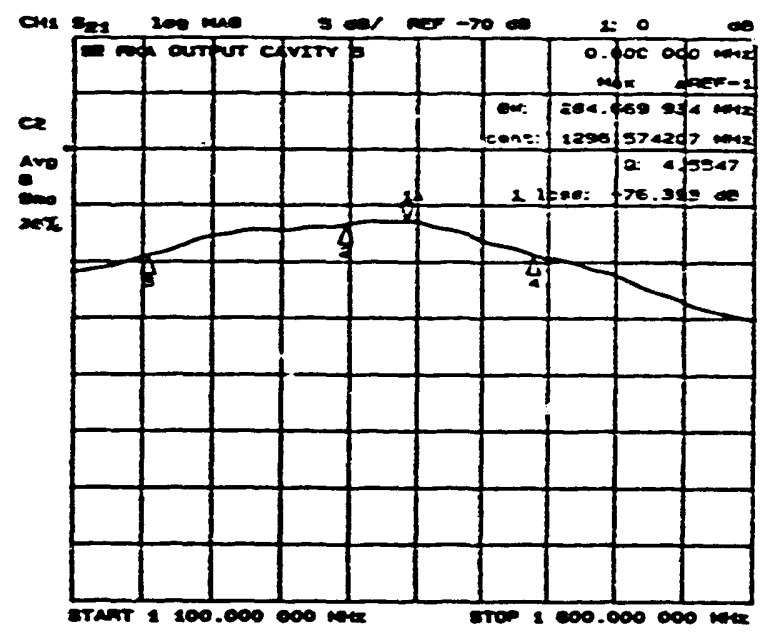

Fig. 9: Cold test of the new output cavity

The new cavity has been installed and is presently being tested at low power. The lower $Q$ of the new output cavity has dropped the gain of the RKA to about $26 \mathrm{~dB}$. Some of this gain loss will be recoverable by tuning the idler closer to the operating frequency. We will gradually increase the drive power to condition the output cavity for operation at full power within the next two months.

\section{SUMMARY}

Peak powers approaching $500 \mathrm{MW}$ at $1.3 \mathrm{GHz}$ have been produced in pulses of 1 $\mu s$ nominal baseline-to-baseline duration. The half power pulse width is $0.5 \mu$ s. These pulses contain an energy of about $160 \mathrm{~J}$. Rf output begins on the rising portion of the current pulse and terminates just before the highest part of the pulsed power curve is reached.

Three dimensional cavity modeling has proven to be critical for designing the very low $Q$ output cavity needed for converting the low impedance modulated electron beam to microwaves. The thrust will be to increase the output power to $1 \mathrm{GW}$ and to widen the pulse to $1 \mu$ s.

\section{ACKNOWLEDGMENTS}

This work was supported and funded jointly by the DOE, the Army Research Laboratory, and the Air Force Phillips Laboratory. 


\section{REFERENCES}

1. M. F. icdman, J. Krall, Y. Y. Lau, and V. Serlin. "Externally modulated intense relativistic electron beams," J. Appl. Phys., Vol.64, no.7, pp. 3353-3379, 1988.

2. M. V. Fazio. W. B. Haynes, B. E. Carlsten, R. J. Faehl, T. J. T. Kwan, and R. M. Stringfield. "Experimental progress on a one microsecond, one kilojoule per pulse, L-band relativistic klystron", Intense Microwave Pulses, H.E. Brandt, Vol.1872, pp. 71-76, SPIE, Los Angeles, CA, 1993.

3. B. E. Carlsten, R. J. Faehl, M. V. Fazio, W. B. Haynes, and R. M. Stringfield, "Intense Space-Charge Beam Physics Relevant to Relativistic Klystron Amplifiers," Submitted to IEEE Trans. Plasma Sci., 1994.

4. HFSS, the High Frequency Structure Simulator marketed by Hewlett-Packard. 

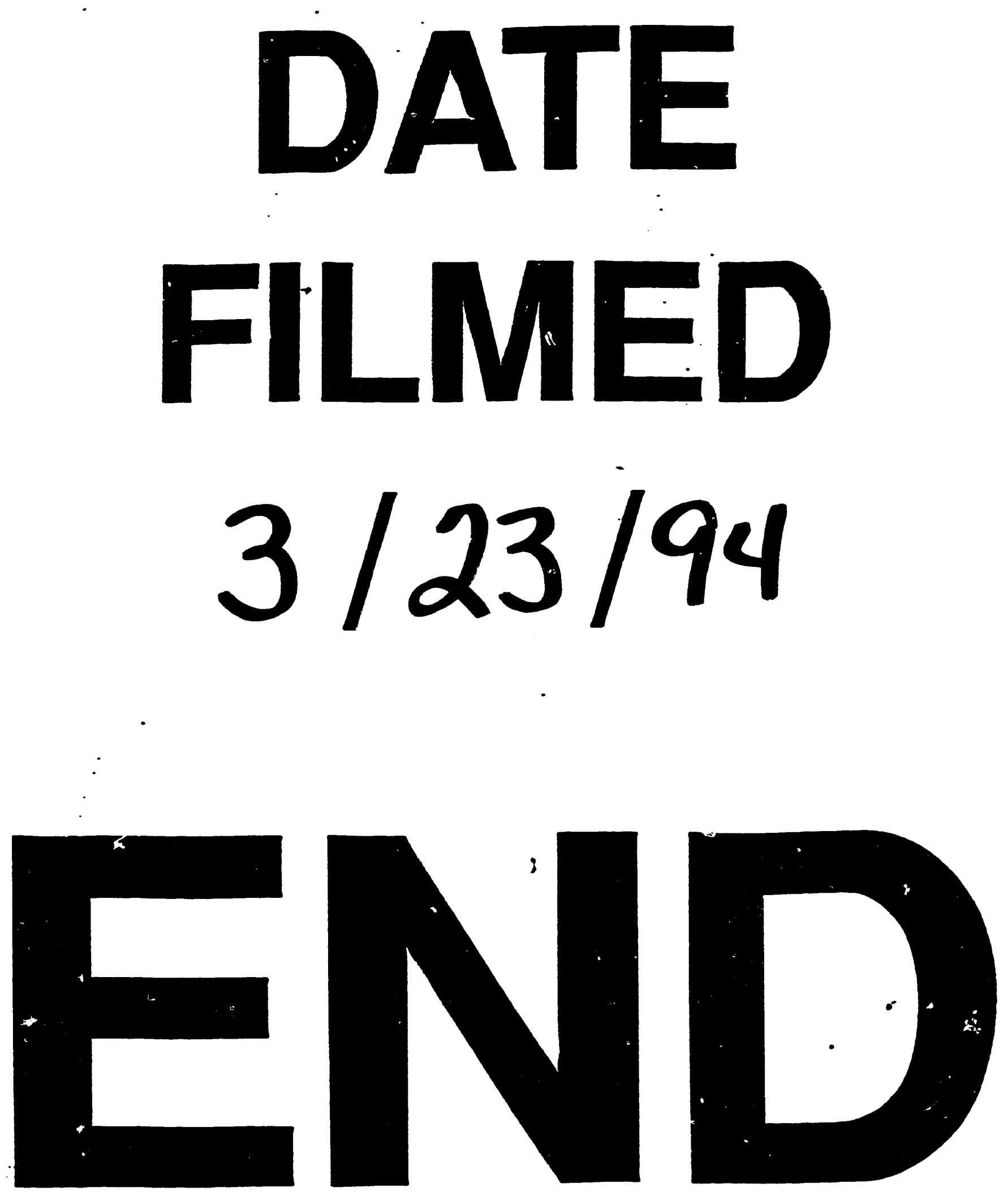